\title{
Unification of Gravitational and Electromagnetic Fields in Curved Space-Time Using Gauge Symmetry of Bianchi Identities
}

\author{
Young Hwan Yun*, Kiho Jang \\ Zero Theoretical Physics Laboratory, Seoul, Republic of Korea \\ Email: *yhyun690@naver.com
}

How to cite this paper: Yun, Y.H. and Jang, K. (2021) Unification of Gravitational and Electromagnetic Fields in Curved SpaceTime Using Gauge Symmetry of Bianchi Identities. Journal of High Energy Physics, Gravitation and Cosmology, 7, 1202-1212. https://doi.org/10.4236/jhepgc.2021.73071

Received: June 11, 2021

Accepted: July 27, 2021

Published: July 30, 2021

Copyright (c) 2021 by author(s) and Scientific Research Publishing Inc. This work is licensed under the Creative Commons Attribution International License (CC BY 4.0).

http://creativecommons.org/licenses/by/4.0/

\begin{abstract}
This paper deals with the generalization of the linear theory of the unification of gravitational and electromagnetic fields using 4-dimensional gauge symmetry in order to solve the contradictions from the Kaluza-Klein theory's unification of the gravitational and electromagnetic fields. The unification of gravitational and electromagnetic fields in curved space-time starts from the Bianchi identity, which is well known as a mathematical generalization of the gravitational equation, and by using the existing gauge symmetry condition, equations for the gravitational and electromagnetic fields can be obtained. In particular, the homogeneous Maxwell's equation can be obtained from the first Bianchi identity, and the inhomogeneous Maxwell's equation can be obtained from the second Bianchi identity by using Killing's equation condition of the curved space-time. This paper demonstrates that gravitational and electromagnetic fields can be derived from one equation without contradiction even in curved space-time, thus proving that the 4-dimensional metric tensor using the gauge used for this unification is more complete. In addition, geodesic equations can also be derived in the form of coordinate transformation, showing that they are consistent with the existing equations, and as a result, they are consistent with the existing physical equations.
\end{abstract}

\section{Keywords}

Bianchi Identity, Killing's Equation, Maxwell's Equation, Gravity, Kaluza-Klein Theory, Unification, Gauge Symmetry, Relativity

\section{Introduction}

After Einstein explained gravity as a geometry based on Newton's equivalence principle through the theory of general relativity in 1915, many scientists in- 
cluding Einstein himself have studied the unified field theory, a new theory encompassing electromagnetic and gravitational phenomena. In 1918 Hermann Weyl, a mathematician, first published the unification theory of general relativity and electromagnetic phenomena [1]. In his unified field theory, Weyl identified electrons as substances continuously distributed in space and tried to unify gravity and electromagnetic forces by expanding them into multidimensional spaces (such as Riemann geometry or four-dimensional space) using a method currently called gauge transformation. However, Einstein rejected Weyl's result and studied the unified field theory in his own way, but did not solve the problem. Kaluza and Klein also came up with the unification theory of gravity and electromagnetic force in 1921 and 1926, which is now leading to string theory [2] [3]. Even though it made cornerstone for the later multi-dimension theories and gave much inspiration to physicists for the dream of unification theory, it has not been established as a complete theory. The main reason for this is that the Kaluza-Klein theory uses a 5-dimensional metric tensor, resulting in various contradictions. Recently, a paper saying that gravitational and electromagnetic field can be unified even in the fourth dimension has been published [4]. It is consistent with existing physical phenomena and there is no mathematical contradiction, but it still has a limitation of the linear approximated space-time. This paper is expanded to show that it can be unified even in a 4-dimensional curved space-time. Starting from the Bianchi identities, Maxwell's equation is obtained by using the conditions of the gauge symmetry. Particularly, since the $2^{\text {nd }}$ Bianchi identity is a general form of the gravitational equation, it tells that the gravitational and electromagnetic fields are naturally unified.

Table 1 and Table 2 below introduce Bianchi identities and Maxwell's equations. Both equations can be categorized into 2 types, and we can see some formal similarities by comparing their types. The 2nd Bianchi identity and Maxwell's equations in the curved space-time have the notations of covariant derivatives [5]. We are going to make relations with Bianchi identities and Maxwell's equations.

We also introduce the gauge of metric tensor in a curved space-time by using previously calculated general gauge transformation [6] [7]. The result is (1-1).

$$
g_{j k}^{\prime}(x) \cong g_{j k}(x)-\varepsilon\left(\nabla_{k} \xi_{j}+\nabla_{j} \xi_{k}\right) \text { with } \varepsilon \rightarrow 0
$$

$\xi_{j}$ means the infinitesimal coordinate transformation

$$
x_{j}^{\prime}=x_{j}+\varepsilon \xi_{j} \text { with } \varepsilon \rightarrow 0
$$

Under Killing's equation, the coordinate transformation of the metric tensor is invariant, thus Equation (1-3) can be used. The solution to this equation, $\xi_{j}$ is called the Killing vector [6] [7] [8].

$$
\nabla_{k} \xi_{j}+\nabla_{j} \xi_{k}=0
$$

By using Killing's equation and the gauge symmetry condition representing the conservation property, we would like to show the deriving process of Maxwell's equation in curved space-time in detail. 
Table 1. Types of Bianchi identity.

\begin{tabular}{c|c}
\hline Types & Bianchi identity \\
\hline The first identity & $R_{i j k l}+R_{i k j j}+R_{i j k k}=0$ \\
\hline The second identity & $\nabla_{l} R_{m i j k}+\nabla_{j} R_{m i k l}+\nabla_{k} R_{m i l j}=0$ \\
& Or $\nabla^{j}\left(R_{j k}-\frac{1}{2} g_{j k} R\right)=0$ \\
& Or $R_{j k}-\frac{1}{2} g_{j k} R=k T_{j k}, \nabla^{j} T_{j k}=0$ \\
\hline
\end{tabular}

(Where $\nabla_{k}$ : covariant derivative, $\partial_{k}$ : ordinary derivative).

Table 2. Types of Maxwell's equations.

\begin{tabular}{c|c|c}
\hline Types & Linear approximated space-time & Curved space-time \\
\hline Homogeneous & $\partial_{k} F_{l i}+\partial_{i} F_{k l}+\partial_{l} F_{i k}=0$ & $\nabla_{k} F_{l i}+\nabla_{i} F_{k l}+\nabla_{l} F_{i k}=0$ \\
\hline Inhomogeneous & $J^{i}=\partial_{j} F^{i j}$ & $J^{i}=\nabla_{j} F^{i j}$ \\
\hline
\end{tabular}

\section{Derivation of Homogeneous Maxwell's Equation from the First Bianchi Identity}

The first Bianchi identity is as follows [6] [9].

$$
R_{i j k l}+R_{i k l j}+R_{i l j k}=0
$$

where

$$
R_{i j k l}=\partial_{k} \Gamma_{i j l}-\partial_{l} \Gamma_{i j k}+\Gamma_{j l}^{p} \Gamma_{i p k}-\Gamma_{j k}^{p} \Gamma_{i p l}
$$

where

$$
\Gamma_{i j l}=\frac{1}{2}\left(\partial_{j} g_{i l}+\partial_{l} g_{i j}-\partial_{i} g_{j l}\right)
$$

When Equation (2-3) is changed as gauge-transform, the metric tensor $g_{j k}$ is replaced with $g_{j k}^{\prime}$ and Equation (1-1) can be used in Equation (2-3). If we intend to check the gauge symmetry, only gauge term $\varepsilon\left(\nabla_{j} \xi_{k}+\nabla_{k} \xi_{j}\right)$ can be substituted into Equation (2-3) and we have Equation (2-4).

$$
\Gamma_{i j l}=\frac{1}{2} \varepsilon\left(\partial_{j}\left(\nabla_{l} \xi_{i}+\nabla_{i} \xi_{l}\right)+\partial_{l}\left(\nabla_{j} \xi_{i}+\nabla_{i} \xi_{j}\right)-\partial_{i}\left(\nabla_{j} \xi_{l}+\nabla_{l} \xi_{j}\right)\right)
$$

When Equation (2-4) is put into the Riemann tensor Equation (2-2), the 3rd and the 4 th terms of right side can be ignored because they are $\mathrm{O}\left(\varepsilon^{2}\right)$ and $\varepsilon$ is infinitesimal. For convenience, $1 / 2$ is omitted and the 1st and 2nd terms of right side of Equation (2-2) are expanded like Equation (2-5).

$$
R_{i j k l}=\partial_{k} \Gamma_{i j l}-\partial_{l} \Gamma_{i j k}=\partial_{k}\left(\partial_{j} g_{i l}+\partial_{l} g_{i j}-\partial_{i} g_{j l}\right)-\partial_{l}\left(\partial_{j} g_{i k}+\partial_{k} g_{i j}-\partial_{i} g_{j k}\right)
$$

When the indexes are rotated, we have Equation (2-6) and Equation (2-7).

$$
\begin{gathered}
R_{i k l j}=\partial_{l} \Gamma_{i k j}-\partial_{j} \Gamma_{i k l}=\partial_{l}\left(\partial_{j} g_{i k}+\partial_{k} g_{i j}-\partial_{i} g_{j k}\right)-\partial_{j}\left(\partial_{k} g_{i l}+\partial_{l} g_{i k}-\partial_{i} g_{k l}\right) \\
R_{i l j k}=\partial_{j} \Gamma_{i l k}-\partial_{k} \Gamma_{i l j}=\underline{\underline{\partial_{j}\left(\partial_{l} g_{i k}+\partial_{k} g_{i l}-\partial_{i} g_{l k}\right)}-\partial_{k}\left(\partial_{j} g_{i l}+\partial_{l} g_{i j}-\partial_{i} g_{l j}\right)}
\end{gathered}
$$


Therefore, Equation (2-5) is

$$
\begin{aligned}
& R_{i j k l}=\partial_{k} \Gamma_{i j l}-\partial_{l} \Gamma_{i j k}= \\
& \partial_{k}\left(\partial_{j}\left(\nabla_{l} \xi_{i}+\nabla_{i} \xi_{l}\right)+\partial_{l}\left(\nabla_{j} \xi_{i}+\nabla_{i} \xi_{j}\right)-\partial_{i}\left(\nabla_{j} \xi_{l}+\nabla_{l} \xi_{j}\right)\right) \\
& -\partial_{l}\left(\partial_{j}\left(\nabla_{k} \xi_{i}+\nabla_{i} \xi_{k}\right)+\partial_{k}\left(\nabla_{i} \xi_{j}+\nabla_{j} \xi_{i}\right)-\partial_{i}\left(\nabla_{i} \xi_{j}+\nabla_{j} \xi_{k}\right)\right)
\end{aligned}
$$

Equation (2-6) is

$$
\begin{aligned}
& R_{i k l j}=\partial_{l} \Gamma_{i k j}-\partial_{j} \Gamma_{i k l}= \\
& \partial_{l}\left(\partial_{j}\left(\nabla_{k} \xi_{i}+\nabla_{i} \xi_{k}\right)+\partial_{k}\left(\nabla_{i} \xi_{j}+\nabla_{j} \xi_{i}\right)-\partial_{i}\left(\nabla_{i} \xi_{j}+\nabla_{j} \xi_{k}\right)\right) \\
& -\partial_{j}\left(\partial_{k}\left(\nabla_{l} \xi_{i}+\nabla_{i} \xi_{l}\right)+\partial_{l}\left(\nabla_{k} \xi_{i}+\nabla_{i} \xi_{k}\right)-\partial_{i}\left(\nabla_{k} \xi_{l}+\nabla_{k} \xi_{k}\right)\right)
\end{aligned}
$$

Equation (2-7) is

$$
\begin{aligned}
& R_{i l j k}=\partial_{j} \Gamma_{i l k}-\partial_{k} \Gamma_{i l j}= \\
& \frac{\partial_{j}\left(\partial_{k}\left(\nabla_{l} \xi_{i}+\nabla_{i} \xi_{l}\right)+\partial_{l}\left(\nabla_{k} \xi_{i}+\nabla_{i} \xi_{k}\right)-\partial_{i}\left(\nabla_{k} \xi_{l}+\nabla_{k} \xi_{k}\right)\right)}{-\partial_{k}\left(\partial_{j}\left(\nabla_{l} \xi_{i}+\nabla_{i} \xi_{l}\right)+\partial_{l}\left(\nabla_{j} \xi_{i}+\nabla_{i} \xi_{j}\right)-\partial_{i}\left(\nabla_{j} \xi_{l}+\nabla_{l} \xi_{j}\right)\right)}
\end{aligned}
$$

According to Equation (2-1), when all the above three equations from Equation (2-8) to Equation (2-10) are added, we can see that the underlined terms of the same types disappear by each other and the final result becomes 0 . Since only the gauge terms are used in the Bianchi identity, it can be said that the $1^{\text {st }}$ Bianchi identity equation is gauge symmetric.

Next, we will show that there are identities between the gauge terms as well. To calculate it, we put all the terms into Table 3 and organize them.

\begin{tabular}{|c|c|c|c|c|c|}
\hline$\partial_{k} \partial_{j}\left(\nabla_{l} \xi_{i}+\nabla_{i} \xi_{l}\right)$ & $\partial_{k} \partial_{l}\left(\nabla_{j} \xi_{i}+\nabla_{i} \xi_{j}\right)$ & $-\partial_{k} \partial_{i}\left(\nabla_{j} \xi_{l}+\nabla_{l} \xi_{j}\right)$ & $-\partial_{l} \partial_{j}\left(\nabla_{k} \xi_{i}+\nabla_{i} \xi_{k}\right)$ & $-\partial_{l} \partial_{k}\left(\nabla_{i} \xi_{j}+\nabla_{j} \xi_{i}\right)$ & $\partial_{l} \partial_{i}\left(\nabla_{i} \xi_{j}+\nabla_{j} \xi_{k}\right)$ \\
\hline$\underline{(1,2)}$ & $\underline{(3,4)}$ & $\underline{(5,6)}$ & $(7,8)$ & $(9,10)$ & $(11,12)$ \\
\hline$\partial_{l} \partial_{j}\left(\nabla_{k} \xi_{i}+\nabla_{i} \xi_{k}\right)$ & $\partial_{l} \partial_{k}\left(\nabla_{i} \xi_{j}+\nabla_{j} \xi_{i}\right)$ & $-\partial_{l} \partial_{i}\left(\nabla_{i} \xi_{j}+\nabla_{j} \xi_{k}\right)$ & $-\partial_{j} \partial_{k}\left(\nabla_{l} \xi_{i}+\nabla_{i} \xi_{l}\right)$ & $-\partial_{j} \partial_{l}\left(\nabla_{k} \xi_{i}+\nabla_{i} \xi_{k}\right)$ & $\partial_{j} \partial_{i}\left(\nabla_{k} \xi_{l}+\nabla_{k} \xi_{k}\right)$ \\
\hline$(13,14)$ & $(15,16)$ & $(17,18)$ & $\underline{\underline{(19,20)}}$ & $\underline{\underline{(21,22)}}$ & $\underline{\underline{(23,24)}}$ \\
\hline$\partial_{j} \partial_{k}\left(\nabla_{l} \xi_{i}+\nabla_{i} \xi_{l}\right)$ & $\partial_{j} \partial_{l}\left(\nabla_{k} \xi_{i}+\nabla_{i} \xi_{k}\right)$ & $-\partial_{j} \partial_{i}\left(\nabla_{k} \xi_{l}+\nabla_{k} \xi_{k}\right)$ & $-\partial_{k} \partial_{j}\left(\nabla_{l} \xi_{i}+\nabla_{i} \xi_{l}\right)$ & $-\partial_{k} \partial_{l}\left(\nabla_{j} \xi_{i}+\nabla_{i} \xi_{j}\right)$ & $\partial_{k} \partial_{i}\left(\nabla_{j} \xi_{l}+\nabla_{l} \xi_{j}\right)$ \\
\hline$\underline{\underline{(25,26)}}$ & $\underline{\underline{(27,28)}}$ & $\underline{\underline{(29,30)}}$ & $\underline{(31,32)}$ & $\underline{(33,34)}$ & $\underline{(35,36)}$ \\
\hline
\end{tabular}

Each element in Table 3 is assigned to two numbers for each term. We search and rearrange pairs in the form like $\partial_{j} \partial_{i} \nabla_{k} \xi_{l}-\partial_{l} \partial_{j} \nabla_{k} \xi_{i}$ with only indexes change. Table 4 is not the unique combination in finding pairs game, but just one of examples.

As previously shown, addition of all values in Table 5 results in 0 , thus

$$
\begin{aligned}
& \partial_{j}\left(\nabla_{k} F_{i l}+\nabla_{i} F_{l k}+\nabla_{k} F_{l i}+\nabla_{i} F_{k l}+\nabla_{l} F_{i k}+\nabla_{l} F_{k i}\right) \\
& +\partial_{l}\left(\nabla_{i} F_{j k}+\nabla_{j} F_{k i}+\nabla_{k} F_{j i}+\nabla_{k} F_{i j}+\nabla_{i} F_{k j}+\nabla_{j} F_{i k}\right) \\
& +\partial_{k}\left(\nabla_{j} F_{l i}+\nabla_{i} F_{l j}+\nabla_{i} F_{j l}+\nabla_{l} F_{i j}+\nabla_{l} F_{j i}+\nabla_{j} F_{i l}\right)=0
\end{aligned}
$$

Table 3. Set of gauge terms for Equation (2-8), Equation (2-9) and Equation (2-10). 
Table 4. Matched gauge terms and position number.

\begin{tabular}{c|c|cc}
\hline$\partial_{j} \partial_{i} \nabla_{k} \xi_{l}-\partial_{l} \partial_{j} \nabla_{k} \xi_{i} \quad(23,7)$ & $\partial_{j} \partial_{l} \nabla_{i} \xi_{k}-\partial_{l} \partial_{k} \nabla_{i} \xi_{j} \quad(28,9)$ & $\partial_{l} \partial_{k} \nabla_{j} \xi_{i}-\partial_{k} \partial_{i} \nabla_{j} \xi_{l} \quad(16,5)$ \\
\hline$\partial_{l} \partial_{j} \nabla_{i} \xi_{k}-\partial_{k} \partial_{j} \nabla_{i} \xi_{l} \quad(14,32)$ & $\partial_{k} \partial_{l} \nabla_{j} \xi_{i}-\partial_{l} \partial_{i} \nabla_{j} \xi_{k} \quad(3,18)$ & $\partial_{k} \partial_{l} \nabla_{i} \xi_{j}-\partial_{j} \partial_{k} \nabla_{i} \xi_{l} \quad(4,20)$ \\
\hline$\partial_{l} \partial_{j} \nabla_{k} \xi_{i}-\partial_{j} \partial_{i} \nabla_{k} \xi_{l} \quad(13,29)$ & $\partial_{j} \partial_{l} \nabla_{k} \xi_{i}-\partial_{l} \partial_{i} \nabla_{k} \xi_{j} \quad(27,17)$ & $\partial_{j} \partial_{k} \nabla_{i} \xi_{l}-\partial_{k} \partial_{l} \nabla_{i} \xi_{j} \quad(26,34)$ \\
\hline$\partial_{k} \partial_{j} \nabla_{i} \xi_{l}-\partial_{l} \partial_{j} \nabla_{i} \xi_{k} \quad(2,8)$ & $\partial_{l} \partial_{i} \nabla_{k} \xi_{j}-\partial_{j} \partial_{l} \nabla_{k} \xi_{i} \quad(11,21)$ & $\partial_{k} \partial_{i} \nabla_{l} \xi_{j}-\partial_{j} \partial_{k} \nabla_{l} \xi_{i} \quad(36,19)$ \\
\hline$\partial_{j} \partial_{i} \nabla_{l} \xi_{k}-\partial_{k} \partial_{j} \nabla_{l} \xi_{i} \quad(24,31)$ & $\partial_{l} \partial_{k} \nabla_{i} \xi_{j}-\partial_{j} \partial_{l} \nabla_{i} \xi_{k} \quad(15,22)$ & $\partial_{j} \partial_{k} \nabla_{l} \xi_{i}-\partial_{k} \partial_{i} \nabla_{l} \xi_{j} \quad(25,6)$ \\
\hline$\partial_{k} \partial_{j} \nabla_{l} \xi_{i}-\partial_{j} \partial_{i} \nabla_{l} \xi_{k} \quad(1,30)$ & $\partial_{l} \partial_{i} \nabla_{j} \xi_{k}-\partial_{k} \partial_{l} \nabla_{j} \xi_{i} \quad(12,33)$ & $\partial_{k} \partial_{i} \nabla_{j} \xi_{l}-\partial_{l} \partial_{k} \nabla_{j} \xi_{i} \quad(35,10)$ \\
\hline$\downarrow$ & $\downarrow$ & $\partial^{2}$ & $\partial_{k} \nabla_{j} F_{l i}$ \\
\hline$\partial_{j} \nabla_{k} F_{i l}$ & $\partial_{l} \nabla_{i} F_{j k}$ & $\partial_{k} \nabla_{i} F_{l j}$ & \\
\hline$\partial_{j} \nabla_{i} F_{l k}$ & $\partial_{l} \nabla_{j} F_{k i}$ & $\partial_{k} \nabla_{i} F_{j l}$ & \\
\hline$\partial_{j} \nabla_{k} F_{l i}$ & $\partial_{l} \nabla_{k} F_{j i}$ & $\partial_{k} \nabla_{l} F_{i j}$ & \\
\hline$\partial_{j} \nabla_{i} F_{k l}$ & $\partial_{l} \nabla_{k} F_{i j}$ & $\partial_{k} \nabla_{l} F_{j i}$ & \\
\hline$\partial_{j} \nabla_{l} F_{i k}$ & $\partial_{l} \nabla_{i} F_{k j}$ & $\partial_{k} \nabla_{j} F_{i l}$ & \\
\hline$\partial_{j} \nabla_{l} F_{k i}$ & $\partial_{l} \nabla_{j} F_{i k}$ & & \\
\hline & & &
\end{tabular}

Here, $F_{i l}=\partial_{i} \xi_{l}-\partial_{l} \xi_{i}$.

Table 5. Bundle with identities.

\begin{tabular}{|c|c|c|c|}
\hline & $\partial_{j}$ & $\partial_{l}$ & $\partial_{k}$ \\
\hline The $1^{\text {st }}$ bundle & $\begin{array}{c}1 \\
\nabla_{k} F_{i l}+\nabla_{k} F_{l i}=0 \\
\nabla_{i} F_{l k}+\nabla_{i} F_{k l}=0 \\
\nabla_{l} F_{k i}+\nabla_{l} F_{i k}=0\end{array}$ & $\begin{array}{c}1 \\
\begin{array}{l}(2) \\
\nabla_{i} F_{j k}+\nabla_{i} F_{k j}\end{array}=0 \\
\nabla_{k} F_{i j}+\nabla_{k} F_{j i}=0 \\
\nabla_{j} F_{k i}+\nabla_{j} F_{i k}=0\end{array}$ & $\begin{array}{l}1 \\
\nabla_{i} F_{l j}+\nabla_{i} F_{j l}=0 \\
\nabla_{j} F_{i l}+\nabla_{j} F_{l i}=0 \\
\nabla_{l} F_{j i}+\nabla_{l} F_{i j}=0\end{array}$ \\
\hline The $2^{\text {nd }}$ bundle & $\begin{array}{l}\text { (1) } \nabla_{k} F_{i l}+\nabla_{i} F_{l k}+\nabla_{l} F_{k i}=0 \\
\text { (2) } \nabla_{k} F_{l i}+\nabla_{i} F_{k l}+\nabla_{l} F_{i k}=0\end{array}$ & $\begin{array}{l}\text { (1) } \nabla_{i} F_{j k}+\nabla_{k} F_{i j}+\nabla_{j} F_{k i}=0 \\
\text { (2) } \nabla_{i} F_{k j}+\nabla_{k} F_{j i}+\nabla_{j} F_{i k}=0\end{array}$ & $\begin{array}{l}\text { (1) } \nabla_{i} F_{l j}+\nabla_{j} F_{i l}+\nabla_{l} F_{j i}=0 \\
\text { (2) } \nabla_{i} F_{j l}+\nabla_{j} F_{l i}+\nabla_{l} F_{i j}=0\end{array}$ \\
\hline
\end{tabular}

The six terms in parentheses, headed by $\partial_{j}, \partial_{k}, \partial_{l}$, can be expressed as two types of bundles that form an identity as shown in Table 5 .

In Table 5, the 1st bundle is composed of $\partial_{j}, \partial_{k}, \partial_{l}$ groups, and each group is composed of 3 pairs and also it makes 0 . By rearranging the 1st bundle, we have the 2 nd bundle in which we can have 6 identities naturally.

Every identity in the $2^{\text {nd }}$ bundle is the homogeneous Maxwell's equation. Thus, we can conclude that the homogeneous Maxwell's equation is derived from the $1^{\text {st }}$ Bianchi identity through gauge symmetry.

One thing to note is that the covariant derivative type $\left(\nabla_{k} F_{l i}+\nabla_{i} F_{k l}+\nabla_{l} F_{i k}=0\right)$ is inextricably related to the ordinary differential type $\left(\partial_{k} F_{l i}+\partial_{i} F_{k l}+\partial_{l} F_{i k}=0\right)$ [10]. The covariant type of Equation (2-12) can be expanded into ordinary type.

$$
\begin{gathered}
\nabla_{k} F_{l i}+\nabla_{i} F_{k l}+\nabla_{l} F_{i k}=0 \\
\partial_{k} F_{l i}-\Gamma_{k l}^{m} F_{m i}-\Gamma_{k i}^{m} F_{l m}+\partial_{i} F_{k l}-\Gamma_{i k}^{m} F_{m l}-\Gamma_{i l}^{m} F_{k m} \\
+\partial_{l} F_{i k}-\Gamma_{l i}^{m} F_{m k}-\Gamma_{l k}^{m} F_{i m}=0
\end{gathered}
$$


In Equation (2-13), the terms of the Christoffel symbol disappear because there is a pair that is deleted by each other, so Equation (2-13) becomes Equation (2-14).

$$
\partial_{k} F_{l i}+\partial_{i} F_{k l}+\partial_{l} F_{i k}=0
$$

In other words, the homogeneous Maxwell's equation becomes a mutually necessary and sufficient condition for a curved space-time and a linear approximated space-time.

\section{Derivation of Inhomogeneous Maxwell's Equation from the Second Bianchi Identity}

The general 2nd Bianchi identity is as follows [6] [9].

$$
\nabla_{l} R_{m i j k}+\nabla_{j} R_{m i k l}+\nabla_{k} R_{m i l j}=0
$$

From the contracted type of $2^{\text {nd }}$ Bianchi identity, we can derive the following equation [6] [9] [11].

$$
\begin{gathered}
R^{j k}-\frac{1}{2} g^{j k} R=k T^{j k} \\
\nabla_{j} T^{j k}=0
\end{gathered}
$$

When $k=\frac{8 \pi G}{c^{4}}$ in (3-3), it becomes Einstein's gravitational equation. Therefore, deriving Maxwell's equation from Bianchi identity can be regarded as deriving the electromagnetic equation from the gravitational equation.

Ricci tensor in Einstein's equation and Christoffel symbol are defined as follows,

$$
\begin{gathered}
R_{j k}=\partial_{i} \Gamma_{j k}^{i}-\partial_{j} \Gamma_{i k}^{i}+\Gamma_{i p}^{i} \Gamma_{j k}^{p}-\Gamma_{j p}^{i} \Gamma_{i k}^{p} \\
\Gamma_{j k}^{i}=\frac{1}{2} g^{i l}\left(\partial_{j} g_{k l}+\partial_{k} g_{j l}-\partial_{l} g_{j k}\right)
\end{gathered}
$$

The gauge term in Equation (1-1) has to be substituted into the metric tensor in Equation (3-5). The Christoffel symbol with only the gauge term substituted is as follows.

$$
\Gamma_{j l}^{i}=\frac{1}{2} g^{i k} \varepsilon\left(\partial_{j}\left(\nabla_{k} \xi_{i}+\nabla_{i} \xi_{k}\right)+\partial_{l}\left(\nabla_{j} \xi_{k}+\nabla_{k} \xi_{j}\right)-\partial_{k}\left(\nabla_{j} \xi_{l}+\nabla_{l} \xi_{j}\right)\right)
$$

When (3-6) is substituted into Equation (3-4), the $3 \mathrm{rd}$ and the 4 th terms of right side can be ignored because they are $\mathrm{O}\left(\varepsilon^{2}\right)$ and $\varepsilon$ is infinitesimal and only the $1^{\text {st }}$ and $2^{\text {nd }}$ terms, which are $\mathrm{O}(\varepsilon)$, are considered. Therefore,

$$
\begin{aligned}
R_{j k}= & \frac{1}{2} \partial_{i}\left[g^{i l}\left(\partial_{k}\left(\nabla_{l} \xi_{j}+\nabla_{j} \xi_{l}\right)-\partial_{l}\left(\nabla_{j} \xi_{k}+\nabla_{k} \xi_{j}\right)\right)\right] \\
& -\frac{1}{2} \partial_{j}\left[g^{i l}\left(\partial_{k}\left(\nabla_{l} \xi_{i}+\nabla_{i} \xi_{l}\right)-\partial_{l}\left(\nabla_{i} \xi_{k}+\nabla_{k} \xi_{i}\right)\right)\right]
\end{aligned}
$$

Here, it is used as the condition of the Killing's equation. Once this happens, the left side of Equation (3-7) becomes always 0. In other words, it has gauge symmetry. 


$$
\begin{aligned}
0= & \frac{1}{2}\left[\partial_{i} \partial_{k}\left(\nabla^{i} \xi_{j}+\nabla_{j} \xi^{i}\right)-\partial_{i} \partial^{i}\left(\nabla_{j} \xi_{k}+\nabla_{k} \xi_{j}\right)\right] \\
& -\frac{1}{2}\left[\partial_{j} \partial_{k}\left(\nabla_{l} \xi^{l}+\nabla^{l} \xi_{l}\right)-\partial_{j} \partial^{i}\left(\nabla_{i} \xi_{k}+\nabla_{k} \xi_{i}\right)\right]
\end{aligned}
$$

If $g^{k \lambda}$ is multiplied to both sides,

$$
\begin{aligned}
0= & \frac{1}{2}\left[\partial_{i} \partial^{\lambda}\left(\nabla^{i} \xi_{j}+\nabla_{j} \xi^{i}\right)-\partial_{i} \partial^{i}\left(\nabla_{j} \xi^{\lambda}+\nabla^{\lambda} \xi_{j}\right)\right] \\
& -\frac{1}{2}\left[\partial_{j} \partial^{\lambda}\left(\nabla_{l} \xi^{l}+\nabla^{l} \xi_{l}\right)-\partial_{j} \partial^{i}\left(\nabla_{i} \xi^{\lambda}+\nabla^{\lambda} \xi_{i}\right)\right]
\end{aligned}
$$

When the condition $j=\lambda$ is applied

$$
\begin{aligned}
0= & \frac{1}{2}\left[\partial_{i} \partial^{j}\left(\nabla^{i} \xi_{j}+\nabla_{j} \xi^{i}\right)-\partial_{i} \partial^{i}\left(\nabla_{j} \xi^{j}+\nabla^{j} \xi_{j}\right)\right] \\
& -\frac{1}{2}\left[\partial_{j} \partial^{j}\left(\nabla_{l} \xi^{l}+\nabla^{l} \xi_{l}\right)-\partial_{j} \partial^{i}\left(\nabla_{i} \xi^{j}+\nabla^{j} \xi_{i}\right)\right]
\end{aligned}
$$

Since the $1^{\text {st }}$ and $4^{\text {th }}$ terms are the same and the $2^{\text {nd }}$ and $3^{\text {rd }}$ terms are the same,

$$
0=\partial_{i} \partial^{j}\left(\nabla^{i} \xi_{j}+\nabla_{j} \xi^{i}\right)-\partial_{i} \partial^{i}\left(\nabla_{j} \xi^{j}+\nabla^{j} \xi_{j}\right)
$$

The $2^{\text {nd }}$ and $3^{\text {rd }}$, and the $1^{\text {st }}$ and $4^{\text {th }}$, respectively, are calculated as follows Table 6.

Since the two calculations are the same, it is doubled.

$$
0=2 \partial_{i} \nabla_{j}\left(\partial^{j} \xi^{i}-\partial^{i} \xi^{j}\right)
$$

So

$$
0=\partial_{i} \nabla_{j}\left(\partial^{j} \xi^{i}-\partial^{i} \xi^{j}\right)
$$

A possible case for Equation (3-13) is that $\nabla_{j} \partial^{j}=\partial_{i} \partial^{i}$ and $\partial_{i} \xi^{i}=\nabla_{j} \xi^{j}$. That is to say $\nabla_{j}=\partial_{i}$. This means that space-time is flat not curved. So for the case of curved space-time, the condition must be that $\nabla_{j}\left(\partial^{j} \xi^{i}-\partial^{i} \xi^{j}\right)$ is conserved for the component $i$.

If the value in parentheses is defined as $F^{i j}$

$$
F^{i j} \stackrel{\text { def }}{=} \partial^{j} \xi^{i}-\partial^{i} \xi^{j}
$$

Then, Equation (3-13) is

$$
0=\partial_{i} \nabla_{j} F^{i j}
$$

Table 6. Calculation for each term in Equation (3-11).

\begin{tabular}{c|c}
\hline $\mathbf{2}^{\text {nd }}+\mathbf{3}^{\text {rd }}$ & $\mathbf{1}^{\text {st }}+\mathbf{4}^{\text {th }}$ \\
\hline & $\partial_{i} \partial^{j} \nabla^{i} \xi_{j}-\partial_{i} \partial^{i} \nabla^{j} \xi_{j}$ \\
$\partial_{i} \partial^{j} \nabla_{j} \xi^{i}-\partial_{i} \partial^{i} \nabla_{j} \xi^{j}$ & Exchange of $i$ and $j$ in the first term \\
$=\partial_{i} \nabla_{j}\left(\partial^{j} \xi^{i}-\partial^{i} \xi^{j}\right)$ & $=\partial_{j} \partial^{i} \nabla^{j} \xi_{i}-\partial_{i} \partial^{i} \nabla^{j} \xi_{j}=\partial^{i} \nabla^{j}\left(\partial_{j} \xi_{i}-\partial_{i} \xi_{j}\right)$ \\
& or \\
& $=\partial_{i} \nabla_{j}\left(\partial^{j} \xi^{i}-\partial^{i} \xi^{j}\right)$ \\
\hline
\end{tabular}


If we define $J^{i}$ like Equation (3-16),

$$
J^{i} \stackrel{\text { def }}{=} \nabla_{j} F^{i j}
$$

Equation (3-15) becomes as follow.

$$
\partial_{i} J^{i}=0
$$

We must also show gauge symmetry of $R$ Ricci scalar which is defined as follows.

$$
R=g^{j k} R_{j k}
$$

As we have already showed $R_{j k}$ has gauge symmetry, $R$ also has gauge symmetry, which means that Einstein equation has gauge symmetry. It shows inhomogeneous Maxwell's equation from Equation (3-16) and continuity equation from Equation (3-17). In Table 7, we summarize that first and second Bianchi identities and the corresponding Maxwell's equations are derived from the coordinate-transformed Bianchi identities and gauge symmetry condition.

\section{Comparison with Kaluza-Klein Theory}

The unification of gravitational and electromagnetic field was first proposed by Kaluza. At that time, the proposed metric tensor was as follows [2] [12].

$$
g_{\mu v}=\left(\begin{array}{lllll}
g_{00} & g_{01} & g_{02} & g_{03} & k A_{0} \\
g_{10} & g_{11} & g_{12} & g_{13} & k A_{1} \\
g_{20} & g_{21} & g_{22} & g_{23} & k A_{2} \\
g_{30} & g_{31} & g_{32} & g_{33} & k A_{3} \\
k A_{0} & k A_{1} & k A_{2} & k A_{3} & k
\end{array}\right)
$$

The geodesic equation, for this metric tensor, is

$$
\frac{\mathrm{d}^{2} x^{i}}{\mathrm{~d} \tau^{2}}+\Gamma_{j k}^{i} \frac{\mathrm{d} x^{j}}{\mathrm{~d} \tau} \frac{\mathrm{d} x^{k}}{\mathrm{~d} \tau}=-k F_{j}^{i} \frac{\mathrm{d} x^{j}}{\mathrm{~d} \tau} \frac{\mathrm{d} x^{5}}{\mathrm{~d} \tau}-k g^{j 5} A_{j}^{i} \frac{\mathrm{d} x^{j}}{\mathrm{~d} \tau} \frac{\mathrm{d} x^{k}}{\mathrm{~d} \tau}
$$

The first term on the right side looks just like the familiar Lorentz force term of a charged particle if we identify $k \frac{\mathrm{d} x^{5}}{\mathrm{~d} \tau}$ with the charge to mass ratio $\mathrm{e} / \mathrm{m}$. However as long as the second term $g^{j 5}$ is not all zero, $g_{\mu v}$ cannot become a tensor [12], because it does not satisfy the tensor condition. Afterward, Klein has supplemented and proposed again as follows [3] [12].

$$
g_{\mu v}=\left(\begin{array}{ccccc}
g_{00}+k A_{0} A_{0} & g_{01}+k A_{0} A_{1} & g_{02}+k A_{0} A_{2} & g_{03}+k A_{0} A_{3} & k A_{0} \\
g_{01}+k A_{0} A_{1} & g_{11}+k A_{1} A_{1} & g_{12}+k A_{1} A_{2} & g_{13}+k A_{1} A_{3} & k A_{1} \\
g_{02}+k A_{0} A_{2} & g_{12}+k A_{1} A_{2} & g_{22}+k A_{2} A_{2} & g_{23}+k A_{2} A_{3} & k A_{2} \\
g_{03}+k A_{0} A_{3} & g_{13}+k A_{1} A_{3} & g_{23}+k A_{2} A_{3} & g_{33}+k A_{3} A_{3} & k A_{3} \\
k A_{0} & k A_{1} & k A_{2} & k A_{3} & k
\end{array}\right)
$$

Equation (4-3) can be seen as a more advanced form than Equation (4-1) because it has orthonormality as Equation (4-4) [12].

$$
g^{\mu v} g_{\mu \lambda}=\delta_{\lambda}^{v}
$$


Table 7. Process of deriving maxwell's equation using gauge symmetry.

\begin{tabular}{|c|c|c|c|}
\hline Gauge transformation & Bianchi identity & Bianchi identity /Maxwell's Equation & from \\
\hline \multirow{4}{*}{$\begin{array}{c}x^{\prime \alpha}=x^{\alpha}+\varepsilon \xi^{\alpha} \\
\text { with } \varepsilon \rightarrow 0 \\
g_{j k}^{\prime}\left(x^{\prime}\right) \cong g_{j k}(x)-\varepsilon\left(\nabla_{k} \xi_{j}+\nabla_{j} \xi_{k}\right)\end{array} \rightarrow$} & \multirow[b]{2}{*}{$R_{i j k l}^{\prime}+R_{i k j j}^{\prime}+R_{i j j k}^{\prime}=0$} & $R_{i j k l}+R_{i k k j}+R_{i j j k}=0$ & $(2-1)$ \\
\hline & & $\nabla_{k} F_{l i}+\nabla_{i} F_{k l}+\nabla_{l} F_{i k}=0$ & $(2-12)$ \\
\hline & $\nabla^{j}\left(R_{j k}^{\prime}-\frac{1}{2} g_{j k} R^{\prime}\right)=0$ & $R_{j k}-\frac{1}{2} g_{j k} R=k T_{j k}$ & $(3-2)$ \\
\hline & $\begin{array}{c}\text { or } \\
R_{j k}^{\prime}-\frac{1}{2} g_{j k} R^{\prime}=k T_{j k}, \nabla^{j} T_{j k}=0\end{array}$ & $J^{i} \stackrel{\text { def }}{=} \nabla_{j} F^{i j}$ & $(3-16)$ \\
\hline
\end{tabular}

The geodesic equation, for this metric tensor, is

$$
\begin{gathered}
\frac{\mathrm{d}^{2} x^{i}}{\mathrm{~d} \tau^{2}}+\Gamma_{j k}^{i} \frac{\mathrm{d} x^{j}}{\mathrm{~d} \tau} \frac{\mathrm{d} x^{k}}{\mathrm{~d} \tau}=-k F_{j}^{i} \frac{\mathrm{d} x^{j}}{\mathrm{~d} \tau} \frac{\mathrm{d} x^{5}}{\mathrm{~d} \tau}-k A_{k} F_{j}^{i} \frac{\mathrm{d} x^{j}}{\mathrm{~d} \tau} \frac{\mathrm{d} x^{k}}{\mathrm{~d} \tau} \\
\text { But } A_{k} F_{j}^{i} \text { in the second term on the right side, has no classical correspon- } \\
\text { dence [12]. } \\
\text { However, the metric tensor proposed in this paper is } 4 \times 4 \text {, not } 5 \times 5 \text { format, } \\
\text { which is different from the above Kaluza and Klein's proposals. } \\
\text { According to Equation }(1-1), \text { the gauge-transformed metric tensor is (4-6). } \\
g_{\mu \nu}^{\prime}=\left(\begin{array}{cccc}
g_{00}+\varepsilon\left(\nabla_{0} A_{0}+\nabla_{0} A_{0}\right) & g_{01}+\varepsilon\left(\nabla_{1} A_{0}+\nabla_{0} A_{1}\right) & g_{02}+\varepsilon\left(\nabla_{2} A_{0}+\nabla_{0} A_{2}\right) & g_{03}+\varepsilon\left(\nabla_{3} A_{0}+\nabla_{0} A_{3}\right) \\
g_{01}+\varepsilon\left(\nabla_{1} A_{0}+\nabla_{0} A_{1}\right) & g_{11}+\varepsilon\left(\nabla_{1} A_{1}+\nabla_{1} A_{1}\right) & g_{12}+\varepsilon\left(\nabla_{2} A_{1}+\nabla_{1} A_{2}\right) & g_{13}+\varepsilon\left(\nabla_{3} A_{1}+\nabla_{1} A_{3}\right) \\
g_{02}+\varepsilon\left(\nabla_{2} A_{0}+\nabla_{0} A_{2}\right) & g_{12}+\varepsilon\left(\nabla_{2} A_{1}+\nabla_{1} A_{2}\right) & g_{22}+\varepsilon\left(\nabla_{2} A_{2}+\nabla_{2} A_{2}\right) & g_{23}+\varepsilon\left(\nabla_{2} A_{3}+\nabla_{3} A_{2}\right) \\
g_{03}+\varepsilon\left(\nabla_{3} A_{0}+\nabla_{0} A_{3}\right) & g_{13}+\varepsilon\left(\nabla_{3} A_{1}+\nabla_{1} A_{3}\right) & g_{23}+\varepsilon\left(\nabla_{2} A_{3}+\nabla_{3} A_{2}\right) & g_{33}+\varepsilon\left(\nabla_{3} A_{3}+\nabla_{3} A_{3}\right)
\end{array}\right)
\end{gathered}
$$

However, $\nabla_{k} A_{j}+\nabla_{j} A_{k}=0$.

The geodesic equation, for this metric tensor, is

$$
\frac{\mathrm{d}^{2} x^{\prime i}}{\mathrm{~d} \tau^{2}}+\Gamma_{j k}^{\prime i} \frac{\mathrm{d} x^{\prime j}}{\mathrm{~d} \tau} \frac{\mathrm{d} x^{\prime k}}{\mathrm{~d} \tau}=0
$$

$\Gamma_{j k}^{\prime i}$ is as follows [6].

$$
\Gamma_{j k}^{\prime i}=\frac{\partial x^{\prime i}}{\partial x^{v}} \frac{\partial x^{\sigma}}{\partial x^{\prime j}} \frac{\partial x^{\tau}}{\partial x^{\prime k}} \Gamma_{\sigma \tau}^{v}+\frac{\partial x^{\prime i}}{\partial x^{v}} \frac{\partial^{2} x^{v}}{\partial x^{\prime j} \partial x^{\prime k}}
$$

And $x_{j}^{\prime}$ has the same definition of Equation (1-2).

Since the geodesic equation proposed as a metric tensor in this paper is the form of coordinate transformation, so the problems that appeared in the Kaluza-Klein theory are not found while maintaining the existing forms.

In Equation (1-1), $\xi$ was used as the gauge term. And it was shown to be in the form of Maxwell's equation when it has gauge symmetry in the gravitational equation. This means that gravitational field and electromagnetic field are completely independent of each other, which is consistent with reality.

On the other hand, deploying 5-dimensional theory has met several problems including the following.

For example, Kaluza introduced the "cylinder condition" hypothesis saying that no component of the 5-dimensional metric depends on the fifth dimension. This extra degree of freedom led that the field equations of fully variable 5-dimensional 
relativity grow enormously in complexity. Afterward, in 1926, Oskar Klein gave Kaluza's classical 5-dimensional theory a quantum interpretation introducing the hypothesis that the fifth dimension was curled up and microscopic, to explain the cylinder condition. He suggested that the geometry of the extra fifth dimension could take the form of a circle, with the radius of $10^{-30} \mathrm{~cm} \mathrm{[13]} \mathrm{[14]}$ [15]. However, it is impossible to explain what kind of energy can make space and time bent so enormously. For another example, according to the Kaluza-Klein theory, they got the electron mass $m \approx 3 \times 10^{30} \mathrm{MeV}$, largely different from the experimentally known $m=0.51 \mathrm{MeV}$. This encountered serious problem obtaining the right electron charge and mass ratio [14] [16].

On the contrary, our theory which is implemented only in 4 dimensions, does not have these kinds of problems.

\section{Conclusion}

The unification of the gravitational and electromagnetic fields in the linearlyapproximated space-time which was shown previously has been also shown even in a curved space-time as well. From the first Bianchi identity, inhomogeneous Maxwell equation is derived, and from the second Bianchi identity homogeneous Maxwell's equation is derived, both by using gauge symmetry condition. Since the second Bianchi identity is equivalent to Einstein's equation, it can be interpreted as the unification of gravitational and electromagnetic fields. The contradiction for the tensor feature and for the geodesic equation which is existing in Kaluza-Klein's theory is cleared by expanding the equations using $4 \times 4$ metric tensor. Even though two fields, gravitational and electromagnetic fields, can be unified, both are completely independent of each other without the causality problem.

\section{Acknowledgements}

I would like to appreciate all of the following people who have helped to complete this paper.

Professor Yoon-Myoung Gimm of Dankook University and Professor Yong Kiel Sung of Dongguk University helped with proofreading and logical development of the thesis.

Professor YoungPak Lee of Hanyang University encouraged and advised to submit the thesis.

Professor Kook-Hee Kang of Sungkyunkwan University has always been interested in our study and introduced the related professors.

Gap-Su Lee, the author of "Aha! General Theory of Relativity" corrected some formulas and improved the quality of the thesis.

\section{Conflicts of Interest}

The authors declare no conflicts of interest regarding the publication of this paper. 


\section{References}

[1] Weyl, H. (1929) Elektron und Gravitation I. Zeitschrift für Physik, 56, 330-352. https://doi.org/10.1007/BF01339504

[2] Theodor, K. (1921). Zum Unitätsproblem in der Physik. Sitzungsberichte der Preussischen Akademie der Wissenschaften. Proceedings of the Prussian Academy of Sciences, Berlin, 1918, 966-972.

[3] Oskar, K. (1926). Quantentheorie und fünfdimensionale Relativitätstheorie. Zeitschrift für Physik, 37, 895-906. https://doi.org/10.1007/BF01397481

[4] Yun, Y., Jang, K. and Sung, Y. (2021) Unification of Gravitational and Electromagnetic Fields Using Gauge Symmetry. Journal of High Energy Physics, Gravitation and Cosmology, 7, 344-351.

[5] Red'kov, V.M., Tokarevskaya, N.G., Bychkouskaya, E.M. and Spix, G.J. (2007) Maxwell Equations in Riemannian Space-Time, Geometrical Modeling of Medias. Nonlinear Dynamics and Applications, 14, 126-139.

[6] Ohanian, H. and Ruffini, R. (1994) Gravitation and Spacetime. Second Edition, W.W. Norton \& Company, New York, 334-335+352-354+316.

[7] Bertschinger, E. (2002) Symmetry Transformations, the Einstein-Hilbert Action, and Gauge Invariance. Physics 8.962, Massachusetts Institute of Technology Department of Physics, Cambridge, USA.

[8] Ronald, A., Maurice, B. and Menahem, S. (1975) Introduction to General Relativity. Second Edition, McGraw-Hill, New York.

[9] Lee, G.S. (2019) Aha! General Theory of Relativity. YESM Publishing Co., Ltd., Seoul, 74-76.

[10] El-Salam, F.A.A. (2013) Fractional Approach of Maxwell Equations in the Curved Spacetime. Journla of Taibah University for Science, 7, 173-179. https://doi.org/10.1016/j.jtusci.2013.04.005

[11] Luigi, B. (1902) Sui simboli a quattro indici e sulla curvatura di Riemann. Rendiconti Accademia Nazionaledei Lincei, 11, 3-7

[12] Straub, W.O. (2008) Kaluza Klein Theory. Pasadena, California. http://www.physicsforums.com/attachments/kaluza-klein-straub-pdf.55316/

[13] Ekspong, G. (1991) The Oskar Klein Memorial Lectures Vol. 1: Lectures by C.N. Yang and S. Weinberg. World Scientific Publishing Co., Pte. Ltd., Singapore. https://doi.org/10.1142/1157

[14] Založnik, A. (2012) Kaluza-Klein Theory. Thesis, University of Ljubljana, Ljubljana, Slovenia.

http://reprints.gravitywaves.com/Kaluza-Klein/Zaloznik-2012-Hamber_KaluzaKleinT heory_LjubljanaSeminar4.pdf

[15] Klein, Oskar (1926) The Atomicity of Electricity as a Quantum Theory Law. Nature, 118, 516 p. https://doi.org/10.1038/118516a0

[16] Greene, B. (2003) The Elegant Universe. Second Edition, Vintage Books, New York, $197 \mathrm{p}$. 